\title{
Role of surgery in brainstem gliomas
}

\author{
Aaron Mohanty \\ Division of Neurosurgery, University of Texas Medical Branch at Galveston, 9.208 John Sealy Annex, 301 University Boulevard, \\ Galveston, TX 77555-0517 USA
}

Address for correspondence:

Dr. Aaron Mohanty

Division of Neurosurgery, University

of Texas Medical Branch at

Galveston, Galveston, TX, USA.

Email: aarmohanty@yahoo.com

DOI: $10.4103 / 0028-3886.53258$

For a pediatric neurosurgeon, one of the most frustrating experiences is managing a child with brainstem glioma. With a median survival of less than 12 months, these children are often managed with radiation therapy and sometimes with chemotherapy. Most tumors in the pediatric population are of diffuse variety $(60-75 \%$ of the brainstem tumors) occurring in the pons and only a selective percentage are focal in nature.

Magnetic resonance imaging (MRI) scan has been the diagnostic modality of choice for arriving at a diagnosis in diffuse brainstem gliomas (conventionally classified as more than $2 \mathrm{~cm}$ in diameter in the MRI). The mainstay of treatment of the diffuse pontine lesions has been radiation therapy. Though it transiently improves the neurological function, the median survival is often less than one year. Post-radiation chemotherapy is of uncertain value in these lesions. Administration of chemotherapy prior to the radiotherapy or concomitant radiation and chemotherapy also has not shown to improve the survival..$^{[1]}$ Attempts are being currently made to improve the drug delivery to the tumor cells by altering the blood brain barrier which, however, is still investigational and has to be evaluated for clinical application. The role of stereotactic biopsy has often been questioned in diffuse pontine lesions and is only practiced in atypical cases. Accompanying hydrocephalus has been conventionally treated with insertion of ventriculoperitoneal shunt or more recently with endoscopic third ventriculostomy.

Going against the conventional wisdom, the authors in the article "Surgical considerations for intrinsic brainstem gliomas: proposal of a modification in classification" have considered patients with tumor diameter larger than $2 \mathrm{~cm}$ for surgery if the lesions appeared well-circumscribed in the preoperative imaging. They have suggested a modification of the currently accepted classification which categorizes the intrinsic tumors as "expanding", "infiltrative" and "ventrally exophytic "types. The study compares 40 patients of "expanding" tumors who were operated with 32 patients of "infiltrative" and "ventrally exophytic" types who were treated with radiotherapy. In the author's experience the patients with expanding variety had a higher overall age of presentation, relatively longer duration of symptoms and slightly lesser incidence of pyramidal signs. The surgery was performed by the senior author to avoid a learning curve with the usage of intraoperative evoked potential monitoring, cranial nerve nuclei mapping and elective postoperative ventilation to limit the morbidity and mortality. The patients with Grade II and Grade III lesions were administered postoperative fractionated radiotherapy or stereotactic radiotherapy. The nonoperative group of patients underwent fractionated radiotherapy without tissue diagnosis. The overall outcome was considerably better with the surgical group. A limited follow-up with high dropout rate was evident in both groups limiting the overall value of the study. ${ }^{[2]}$

The article raises several important questions. Is there a role for a debulking or excisional surgery in brainstem gliomas? How radical should one be in excising the lesion? What percentages of tumors are amenable to surgical excision? Do we consider excisional surgery based on the clinical and radiological picture only or 
are histological characteristics also important? Can we recommend excisional surgery as a standard of care for brainstem gliomas?

After going through the article, one would concur with the authors that the role of excisional surgery in brainstem gliomas needs to be revisited. The guidelines formulated a few decades ago obviously were true for those periods and with advanced technology, a selected group of lesions can be excised safely which were otherwise deemed inoperable. As the authors have shown, meticulous surgical techniques with intraoperative monitoring in well-defined and localized lesions are essential for a better postoperative outcome. However, in view of the location, these tumors should always be considered for a "safe excision" avoiding the temptation for an excellent postoperative imaging which is not uncommonly accompanied with a gross morbidity thus limiting the effectiveness of the surgery. Sound clinical judgment and excellent surgical acumen are highly essential in identifying the appropriate tumors for a radical excision and avoiding tumors where similar procedure is associated with undesirable morbidity and mortality.

Considering the demographics, it is apparent that there were more patients in the surgical group than in the nonsurgical group. However, it is important to note that there was a significant age difference between the surgical and nonsurgical group. The mean age of the patients who required surgery was higher (19.2 years) as compared to the mean age of patients who were managed nonsurgically (5.2 years). This correlates well with the results of a previous study of 48 adults with brainstem tumors (mean age of onset 34 years), $46 \%$ had diffuse intrinsic low-grade gliomas with a long pre-diagnosis clinical history. ${ }^{[3]}$ This confirms that relatively benign intrinsic brainstem tumors are more common in the adult population than in children and may be more amenable for surgical resection.
It is also evident from the present study that in twothirds of the patients in the operative group the lesion was low-grade (Grade I and II). Unfortunately, we do not have similar data available for the nonoperative group. Though the past studies have shown that most of the diffuse pontine lesions are malignant, it is not uncommon to find a few low-grade lesions which may be amenable to surgery. In a recent study, of the 24 children who had diffuse pontine gliomas with imaging characteristics suggestive of malignant glioma, two were found to have low-grade lesions by stereotactic biopsy. ${ }^{[4]}$ Does this indicate that the surgeon should be more generous in considering biopsy, especially in cases where the classical radiological appearance is not visualized?

Now, can excisional surgery be recommended as a "standard of care" in selected brainstem gliomas? I would be certainly cautious to recommend excisional surgery as a "standard of care" for these patients. This is because results of the present study can only be repeated with excellent technical skills coupled with experience in a dedicated institutional setup. However, as newer modalities of investigations are available and technical standards continuously improve, new recommendations will certainly soon be available for these once "inoperable lesions".

\section{References}

1. Frazier JL, Lee J, Thomale UW, Noggle JC, Cohen K, Jallo GI. Treatment of diffuse intrinsic brainstem gliomas: Failed approaches and future strategies. J Neurosurg Pediatr 2009;3:259-69.

2. Mehta VS, Chandra PS, Singh PK, Garg A, Rath GK. Surgical considerations for 'brainstem gliomas': Proposal of a modification in classification. Neurol India 2009;57:274-81.

3. Guillam JS, Monjour A, Taillandier L, Devaux B, Varlet P, HaieMeder $\mathrm{C}$, et al. Brainstem gliomas in adults: prognostic factors and classification. Brain 2001;124:2528-39.

4. Roujeau T, Machado G, Garnett MR, Miquel C, Pugret S, Geoerger $\mathrm{B}$, et al. Stereotactic biopsy of diffuse pontine lesions in children. J Neurosurg 2007;107:1-4.

Accepted on 04-05-09

Source of Support: Nil, Conflict of Interest: None declared. 